\title{
Learned helplessness in an appetitive discrete-trial T-maze discrimination test
}

\author{
R. F. S. JOB \\ University of Sydney, Sydney, New South Wales, Australia
}

\begin{abstract}
Four groups of rats were exposed to response-contingent, yoked noncontingent, or en masse food deliveries in a Skinner box or to no experimental pretreatment. All groups were subsequently tested for transfer of the learned-helplessness effect to an appetitive discrete-trial T-maze discrimination employing experimenter-blind procedures. The yoked group showed retarded discrimination learning in comparison with the response-contingent and naive control groups but not in comparison with the en masse control group. This nonsignificant difference between the yoked group and the en masse group may reflect the effect of limited exposure to uncontrollability in the en masse group. The groups did not differ in terms of the speed of maze traversal. The latter result suggests that the learned-helplessness effect observed in discrimination was not due to a competing response.
\end{abstract}

Learned-helplessness theory proposes that the retarded learning often observed after exposure to uncontrollable shock (Brown \& Dixon, 1983; Maier, 1970; Seligman \& Beagley, 1975) is due to the subjects' learning that their responses and the reinforcement are unrelated (Alloy \& Seligman, 1979; Maier \& Jackson, 1979). The major proponents of learned-helplessness theory have also suggested that a similar effect could be caused by exposure to uncontrollable appetitive outcomes, such as food (Maier \& Seligman, 1976; Seligman, 1975). However, the relevant investigations have resulted in inconsistent findings.

In experiments with human subjects, the appetitiveaversive distinction is clouded by the possibility that subjects are responding because of the positive reinforcement (approval) or because of the negative reinforcement (offset of disapproval). In many experiments, both possibilities exist, with both positive ("right") and negative ("wrong") feedback being employed (e.g., Benson \& Kennelly, 1976; Danker-Brown \& Baucom, 1982; Griffith, 1977; Hiroto \& Seligman, 1975; Nation \& Massad, 1978).

Experiments in which pigeons served as subjects in appetitive situations have variously indicated the presence of the learned-helplessness effect (Enberg, Hansen, Welker, \& Thomas, 1972), an effect that lasts only a few trials (Welker, 1976), or no effect (Schwartz, Reisberg, \& Vollmecke, 1974). Although many relevant experiments that employed rats have suffered from such methodological problems as not employing a responsecontingent group (Bainbridge, 1973, Experiments 2 and 3; Mullins \& Winefield, 1977), the full range of results was again obtained: observation of the learned-helplessness effect (Oakes, Rosenblum, \& Fox, 1982; Winefield, 1978), failure to observe the effect (Wheatley, Welker,

The author acknowledges the help of R. F. Porcheron throughout this project. Requests for reprints should be addressed to the author, Psychology Department, University of Sydney, Sydney, N.S.W. 2006, Australia.
\& Miles, 1977 [erratum, 1978]), and facilitation of the subject exposed to uncontrollability (Beatty \& Maki, 1979; Calef et al., 1984; Job, 1986).

The learned-helplessness effect in the appetitive situation has typically been examined in an operant Skinner box situation. In two exceptional studies, opposing results have been obtained: Winefield (1978) employed a discrete-trial two-choice visual discrimination task and supported the occurrence of the learned-helplessness effect, whereas Calef et al. (1984) used a discrete-trial runway task and observed a facilitation effect in the subjects previously exposed to uncontrollability. A possible explanation of these divergent results lies in the measures of learning employed. Possibly, the learned-helplessness effect is observable in a discrimination measure but not in a latency measure. This possibility is not resolved by either experiment, since Calef et al. used no discrimination measures and Winefield reported no latency data. This possibility is predictable from learned-helplessness theory, which clearly allows the prediction that the cognitive deficit will result in impaired discrimination learning. However, in a straight runway, the contingency between the response and the reinforcer may be too easily observed, making the task insensitive to the learnedhelplessness effect in rats (Maier, Albin, \& Testa, 1973; Maier \& Testa, 1975).

The above account of the inconsistency of Calef et al.'s (1984) and Winefield's (1978) findings is tested in the present experiment. The test task involved a complex discrimination in a T-maze employing a discrete-trial procedure. From the preceding analysis, it was predicted that the discrimination-learning results would support the occurrence of the learned-helplessness effect (as in the Winefield experiment), while the latency data would not (as in Calef et al.'s experiment). The latter prediction was based on the extrapolation from the straight runway to the T-maze, since without discrimination, the T-maze involves running with random partial reinforcement. Thus, 
the response of running the maze (without discrimination) may be regarded as having a simple response-reinforcer contingency.

\section{METHOD}

\section{Subjects}

The subjects were 40 male Wistar rats aged 90-100 days at the beginning of the experiment. The rats were housed 3 or 2 per cage as dictated by numbers and were maintained on a 12 -h light-dark cycle with water ad lib.

\section{Apparatus}

Three identical Skinner boxes were used in the pretreatment phase. They measured $22.5 \times 33.5 \times 32.5 \mathrm{~cm}$. The walls were constructed of clear Plexiglas, blackened on the outside, and the floors were stainless steel. To one end wall was attached a food cup $2.5 \mathrm{~cm}$ in diameter and located $3 \mathrm{~cm}$ above the floor and $6 \mathrm{~cm}$ from the left side wall. Located $9 \mathrm{~cm}$ above the floor and immediately to the right of the food cup was a $10-\mathrm{cm}$-long, 2-cm-wide Plexiglas bar. Barpress responses were automatically detected and recorded using Hales equipment; 45-mg food pellets were delivered by Davis Scientific Instruments pellet dispensers.

The test phase was conducted in a T-maze constructed of black painted wood with clear Plexiglas lids. The inside width of the maze was $10 \mathrm{~cm}$; the start arm was $78 \mathrm{~cm}$ long, including the $23-\mathrm{cm}$ startbox, and each choice arm was $85 \mathrm{~cm}$ long, including a $23-\mathrm{cm}$ goalbox. Photocell units that detected the passing of the rat were located along the maze so that three separate response latencies were measured: time to travel from the startbox to the choice point (henceforth, start-arm latency); time in the choice point, which ended when the rat moved $10 \mathrm{~cm}$ along one of the goal arms (henceforth, choicepoint latency); and time to travel from the choice point to the goalbox (henceforth, goal-arm latency).

The laboratory was illuminated by overhead fluorescent lights and ventilated by a fan, which also provided background noise.

\section{Procedures}

Pretreatment. The pretreatment-phase procedures were those known to produce the learned-helplessness effect in an operant test situation (Job, 1986, Experiment 4).

After 2 days of acclimatization to the laboratory, the rats were placed on a 23-h food-deprivation schedule of access to wet mash for $1 \mathrm{~h} /$ day. This reduced their weights by approximately $20 \%$ over 14 days prior to the beginning of the experiment. On the 15 th day, each subject was allowed 5 min of adaptation to the Skinner box, with 10 food pellets in the food cup and no manipulanda present.

The subjects were assigned to one of four basic groups in matched sets of 4 subjects on the basis of their predeprivation body weights so that each set of 4 rats consisted of 1 rat from each basic group. The four groups received differential pretreatment as follows:

Response-contingent $(R C)$ group. $\mathrm{RC}$ rats were allowed to earn a total of 980 pellets by barpressing on a continuous reinforcement (CRF) schedule. Seven sessions occurred over an 8-day period, with the rat allowed to earn the following numbers of pellets per session: $40,40,100,200,200,200,200$. In order to avoid a possible selection bias in this group, no subject was discarded for failure to learn; rather, shaping by successive approximation was employed. This was rarely necessary. The subjects were 22 to $23 \mathrm{~h}$ food deprived at the time of the experimental sessions.

Yoked noncontingent $(Y)$ group. Each subject in this group was yoked to an RC subject. Pretreatment for the $Y$ group was identical to that of the RC group, except that the delivery of the 980 pellets was determined by each $Y$ subject's RC partner in the adjoining box, and was thus independent of the $Y$ subject's behavior. The bar was present for the $\mathrm{Y}$ group, as it was for the $\mathrm{RC}$ group. Barpresses had no programmed effect.

En masse control $(M C)$ group. This group was given the pretreatment used by Wheatley et al. (1977). The MC group received the same pretreatment as the RC group except in one respect. The same number of food pellets as would be earned by the RC group in each pretreatment session were present in the food cup at the beginning of the session. The session length for each MC rat was determined by the time taken for the RC rat in the set to earn the required number of pellets. Barpresses had no programmed effect.

Naive control (NC) group. Since, in learned-helplessness experiments, this type of control group is sometimes used instead of the MC group, it was decided to use both groups in the present series of experiments. Any effect attributable to the different control groups would thus be identifiable. This group did not receive Skinner box pretreatment. NC rats were kept in the home cage during the seven pretreatment sessions, receiving an additional amount of Purina rat cubes to equal the pellets received by the other groups. These foods are known to be of similar caloric value (Valle, 1969).

Re-marking. On the day between pretreatment and testing, a second experimenter rearranged the identifying marks on the rats according to a code known only to that experimenter. The home cages were also reordered to eliminate any clues for identification that the test experimenter might derive from this source (e.g., the heaviest rat in cage 2 belongs to the RC group). In this way, the author conducted both the pretreatment and the test phases but during the test was unaware of the pretreatment received by any particular rat.

Test. Testing began $48 \mathrm{~h}$ after the last pretreatment session and was identical for all groups. The rats were required to learn to alternate their responses-right, left, right, and so on. So, for example, after the first (reinforced) right turn, no further right turns were reinforced until eventually a left turn occurred. This left turn would be reinforced, and so on. This procedure does not allow a simple spatial preference to be maintained on a partial reinforcement schedule, since only the first trial of a series of consecutive turns to one side could be reinforced.

All rats were given a minimum of 60 noncorrection trials, with training continued to the criterion of five consecutive correct responses. Ten trials occurred each day with an intertrial interval of approximately $3 \mathrm{~min}$.

For half the subjects in each group, the first response was reinforced; for the other half, it was not. This was achieved by placing the food in both goalboxes for half the subjects, and in neither goalbox for the others. This procedure allowed half the rats in each group to receive their first reinforcement on their initially preferred side; the other half received their first reinforcement on their apparently nonpreferred side. Reinforcement consisted of four food pellets.

\section{RESULTS}

For all statistical tests, alpha was set at .05 .

\section{Latency Measures}

A reciprocal transformation was performed on all the latency data before analysis, since the raw data were not normally distributed in terms of both skewness and kurtosis. A two-way (4 groups $\times 60$ trials) analysis of variance was applied to each of the four speed measures (the reciprocals of start-arm latency, choice latency, goal-arm latency, and total latency). The results of these analyses were similar for all four measures: in no case was the 


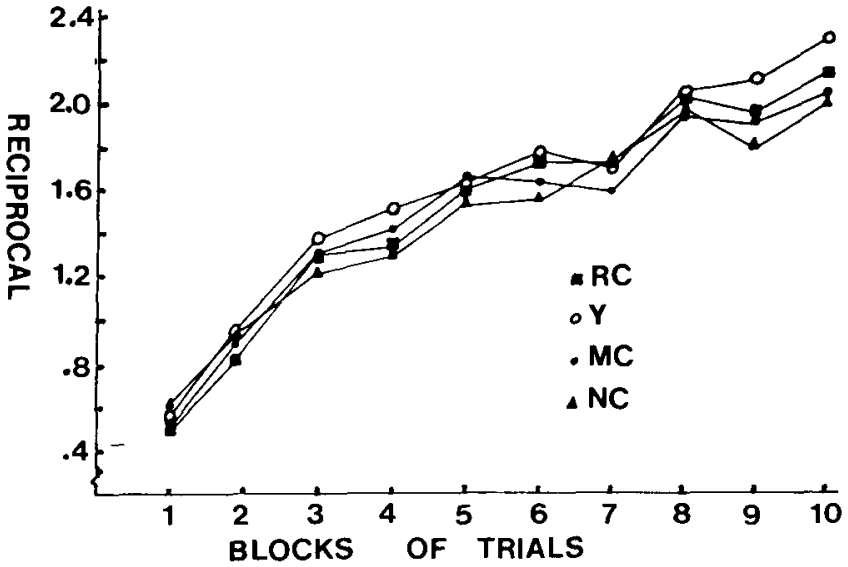

Figure 1. Reciprocal of total latency (in seconds) to traverse the T-maze as a function of blocks of six trials.

groups effect or the groups $\times$ trials interaction significant, with $F$ values ranging from .96 to .08 . As would be expected, in all four cases the trials effect was significant $[F \mathrm{~s}(59,531)=47.32,26.48,51.31$, and 42.53 ; $p<.001$ in all cases]. Since the results of these measures were very similar, only the results for the total latency measure are presented in Figure 1, which clearly indicates improvement over trials.

\section{Discrimination}

Discrimination learning was measured in terms of trials to reach the criterion of five consecutive correct trials. In order to ensure that criterion had not been reached simply by chance responding rather than learning, the alternation (correct choice) rates for each rat on Day 1 and Day 6 were compared. A significant improvement occurred (binomial test, $Z=4.06, p=.00003$; Siegel, 1956, pp. 36-42).

Because the trials to criterion data were skewed with flat kurtosis, a Walsh test was employed in comparing the $Y$ group with each of the other groups. The Walsh test was employed since it assumes interval measurement and has very high power efficiency (Siegel, 1956, p. 87). The results indicated that the $\mathrm{Y}$ group took significantly more trials to reach criterion than the RC group (means $=65.2$ and $41.2 ; p<.005$ ) or the $\mathrm{NC}$ group (mean $=$ $52.9 ; p<.025)$. However, the $\mathrm{Y}$ and MC control groups did not differ significantly (mean $=58.6 ; p>.05)$. The $\mathrm{RC}$ and NC groups did not differ significantly ( $p>.05$ ), indicating no clear facilitation due to prior experience of controllability.

\section{DISCUSSION}

The discrimination test employed met the two requirements for demonstration of the learned-helplessness effect: first, as evidenced by the significant improvement from Day 1 to Day 6, learning occurred; yet, second, the response-reinforcer contingency was not obvious. The response-reinforcer contingency must not be obvious if the learned-helplessness effect is to be produced in rats (Maier et al., 1973; Maier \& Seligman, 1976; Maier \& Testa, 1975). Despite the apparently appropriate nature of the task, the learned-helplessness effect was not fully confirmed in that the yoked and en masse control groups did not differ significantly. However, the yoked group did show retarded discrimination learning in comparison with the response-contingent and naive control groups. In almost all previously reported experiments, these two comparisons alone are regarded as sufficient to confirm the operation of the learned-helplessness effect. In particular, the experiments conducted in the appetitive situation with a discrimination test have not employed a control group given en masse food-pellet deliveries during pretreatment (e.g., Bainbridge, 1973; Mullins \& Winefield, 1977; Winefield, 1978). Consequently, without the en masse group, the present experiment confirms the occurrence of the learned-helplessness effect.

The en masse group may be regarded as appropriate, since it has the advantage of exposure to the apparatus and the food without experience of controllability, whereas the naive control group does not receive the food and the response-contingent group does experience controllability. Nonetheless, the en masse group suffers from limited exposure to uncontrollability: the en masse food represents exposure to uncontrollability on each of the seven pretreatment sessions. Furthermore, this is added to the food-deprivation schedule experienced by all groups. The deprivation procedure of allowing access to food once a day may also be seen as an example of uncontrollability. Thus, the en masse group may be viewed as receiving a minimum of 30 exposures to noncontingent food deliveries during the experiment (including 7 pretreatment sessions and 23 food-deprivation feedings), which is more exposure to uncontrollability than that received by the response-contingent and naive control groups, yet less than that received by the yoked group. Furthermore, the en masse group received uncontrollability in the experimental apparatus in the laboratory. This may explain the nonsignificant difference between the en masse and the yoked groups in the present experiment, and the limited support found for the learnedhelplessness effect in the other experiments that included an en masse group and found no difference between that group and the response-independent group (Job, 1986; Wheatley et al., 1977 [erratum, 1978]).

Most experiments that have induced learned helplessness in an appetitive operant situation have also tested for learned helplessness in an operant situation (e.g., Enberg et al., 1972; Oakes et al., 1982; Schwartz et al., 1974; Welker, 1976; Wheatley et al., 1977). On the other hand, experiments that have employed discrimination testing for appetitive learned helplessness also provided pretreatment in a discrimination situation with subjects exposed to "unsolvable problems" (Bainbridge, 1973; Mullins \& Winefield, 1977; Winefield, 1978). The present experiment, with operant pretreatment, represents a clear example of transfer of the learned-helplessness effect from 
an appetitive operant to an appetitive discrete-trial discrimination situation.

The exception to the above analysis appears to be the experiment reported by Calef et al. (1984), who examined a similar transfer from operant pretreatment to a discretetrial test. However, they found that uncontrollability caused no significant effect on subsequent straight-alley running speed in three of the four stages of testing, whereas running was facilitated by prior uncontrollability in the remaining stage. The present results are consistent with these findings: in terms of speed (reciprocal of latency), the yoked group was not debilitated in the present experiment. Because the learned-helplessness effect was supported in terms of the discrimination results, the present results suggest that the findings of Calef et al. arose from the use of a speed measure in testing, not from a failure of transfer of the learned-helplessness effect.

The present study also offers, in a sense, stronger sup port for the learned-helplessness theory than does that of Jackson, Alexander, and Maier (1980), who examined the effect of inescapable shock on Y-maze learning with shock offset reinforcement. Those authors argued that, according to response competition theory, "reduced activity should not produce inaccurate choices, only slow ones" (Jackson et al., 1980, p. 1). In support of learnedhelplessness theory and response competition, they found that inescapable shock led to both slower and less accurate choices. However, the problem arises that the slower running would lead to a greater time delay from the response of making the correct turn and the interruption of the photoelectric beam (which caused the shock offset and was located $12.5 \mathrm{~cm}$ from the choice point). Thus, slower running would lead to a greater delay between the choice response and reinforcement, which could itself adversely affect discrimination learning. This potential explanation of the results of Jackson et al. is not applicable to the present experiment, since the yoked group did not run more slowly than the other groups. Because this explanation is not available, the present results present a problem for response competition theory. This difference between the experiments may reflect the potential for shock to result in the freeze response while food presentations do not produce such a response.

\section{Conclusion}

The predictions that the learned-helplessness effect would be supported in the discrimination data but not in the latency data were supported by the results. Transfer of the learned-helplessness effect from an operant to a discrete-trial situation was supported, with experimenterblind procedures being used in the test phase.

\section{REFERENCES}

Alloy, L. B., \& Seligman, M. E. P. (1979). On the cognitive component of learned helplessness and depression. In G. H. Bower (Ed.), The psychology of learning and motivation: Advances in research and theory (Vol. 16). New York: Academic Press.
BaINBridge, P. L. (1973). Learning in the rat: Effect of early experience with an unsolvable problem. Journal of Comparative \& Physiological Psychology, 82, 301-307.

BEATTY, W. W., MAKI, W. S. (1979). Acquisition of instrumental responding following noncontingent reinforcement: Failure to observe "learned laziness" in rats. Bulletin of the Psychonomic Sociery, 13, 268-271

Benson, J. S., \& KenNelly, K. J. (1976). Learned helpiessness: The result of uncontrollable reinforcements or uncontrollable aversive stimuli. Journal of Personality \& Social Psychology, 34, 138-145.

Brown, G. E., \& Dixon, P. A. (1983). Learned helplessness in the gerbil. Journal of Comparative Psychology, 97, 90-92.

Calef, R. S., Metz, R. A., Atkinson, T. L., Pellerzi, R. C., TayLOR, K. S., \& Geller, E. S. (1984). Acquisition of running in the straight alley following experience with response-independent food. Bulletin of the Psychonomic Society, 22, 67-69.

Danker-Brown, P., \& BaUCom, D. H. (1982). Cognitive influences on the development of learned helplessness. Journal of Personality \& Social Psychology, 43, 793-801

EnberG, L. A., Hansen, G., Welker, R. L., \& Thomas, D. R. (1972). Acquisition of key pecking via autoshaping as a function of prior experience: "Learned laziness?' Science, 178, 1002-1004.

Griffith, M. (1977). Effects of noncontingent success and failure on mood and performance. Joumal of Personality, 45, 442-457.

Hiroto, D. S., \& Seligman, M. E. P. (1975). Generality of learned helplessness in man. Journal of Personality \& Social Psychology, 31, 311-327.

Jackson, R. L., Alexander, J. H., \& Maler, S. F. (1980). Learned helplessness, inactivity and associative deficits: Effects of inescapable shock on response choice escape learning. Journal of Experimental Psychology: Animal Behavior Processes, 6, 1-20.

JoB, R. F. S. (1986). Learned helplessness in chickens: A test of the theoretical accounts of the resistance of some subjects to the learned helplessness effect. Manuscript submitted for publication.

MaIER, S. F. (1970). Failure to escape traumatic electric shock: Incompatible skeletal-motor responses or learned helplessness? Learning \& Motivation, 1, 157-169.

Maier, S. F., Albin, R. W., \& Testa, J. J. (1973). Failure to learn to escape in rats previously exposed to inescapable shock depends on nature of escape response. Journal of Comparative \& Physiological Psychology, 85, 581-592.

MAIER, S. F., \& JACKsON, R. L. (1979). Learned helplessness: All of us were right (and wrong): Inescapable shock has multiple effects. In G. H. Bower (Ed.), The psychology of learning and motivation: Advances in research and theory (Vol. 16). New York: Academic Press.

Maier, S. F., \& Seligman, M. E. P. (1976). Learned helplessness: Theory and evidence. Joumal of Experimental Psychology: General, $105,3-46$.

MAIER, S. F., \& TesTA, J. J. (1975). Failure to learn to escape by rats previously exposed to inescapable shock is partly produced by associative interference. Journal of Comparative \& Physiological Psychology, 88, 554-564.

Mullins, G. P., \& Winefield, A. H. (1977). Immunization and helplessness phenomena in the rat in a nonaversive situation. Animal Learning \& Behavior, 5, 281-284.

Nation, J. R., \& MASSAd, P. (1978). Persistence training: A partial reinforcement procedure for reversing learned helplessness and depression. Journal of Experimental Psychology: General, 107, 436-451.

OAKes, W. F., Rosenblum, J. L., \& Fox, P. E. (1982). “Manna from heaven": The effects of noncontingent appetitive reinforcers on learning in rats. Bulletin of the Psychonomic Society, 19, 123-126.

SchWARTZ, B., Reisberg, D., \& Vollmecke, T. (1974). Effects of treadle training on autoshaped keypecking: Learned laziness and learned industriousness or response competition? Bulletin of the Psychonomic Society, 3, 369-372.

Seligman, M. E. P. (1975). Helplessness: On depression, development and death. San Francisco: Freeman, 1975.

Seligman, M. E. P., \& BeAgley, G. (1975). Learned helplessness in the rat. Journal of Comparative \& Physiological Psychology, 88, 534-541. 
Seligman, M. E. P., \& Maier, S. F. (1967). Failure to escape traumatic shock. Journal of Experimental Psychology, 74, 1-9.

SIEGEL, S. (1956). Nonparametric statistics for the behavioral sciences. New York: McGraw-Hill.

VALLE, F. P. (1969). A note on the caloric values of Purina chow and Noyes pellets. Psychonomic Science, 15, 38 .

WELKER, R. L. (1976). Acquisition of a free-operant-appetitive response in pigeons as a function of prior experience with response-independent food. Learning \& Motivation, 7, 394-405.

WheAtley, K. L., Welker, R. L., \& Miles, R. C. (1977). Acquisition of barpressing in rats following experience with responseindependent food. Animal Learning \& Behavior, 5, 236-242. (Erratum, Animal Learning \& Behavior, 1978, 6, 51).
WiNeFIELD, A. H. (1978). The effect of prior random reinforcement on brightness discrimination learning in rats. Quarterly Journal of Experimental Psychology, 30, 113-119.

\section{NOTE}

1. Unfortunately, a three-way analysis of variance with the three latency measures treated together was not possible since, in the repeated measures design, over 1,000 variables and 7,200 data points would be involved. This data set was too large for the computing facilities available. Nonetheless, each analysis of latencies reported is based on 2,400 data points.

(Manuscript received July 17, 1986;

revision accepted for publication December 2, 1986.) 